\title{
SOBRE LA DIMENSIÓN JUSFILOSÓFICA DEL PROCESO
}

\author{
Willis Santiago Guerra Filho \\ Universidad Federal de Ceará (Brasil)
}

\section{La dimensión teorética del proceso}

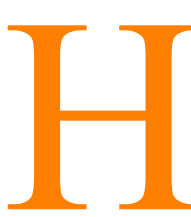

ace relativamente poco tiempo que los teóricos del derecho pasaron a dedicar mayor atención a su aspecto procedimental, antes considerado como poseedor de una función subsidiaria en relación con las normas materiales, portadoras éstas de valoraciones y modelos de conducta, restando para las normas procedimentales el problema meramente técnico de su realización. Autores de la fase tardía del pandectismo alemán, cuando ya se inicia la deducción del Derecho Civil de base romanista de una teoría general del derecho, proclaman en el último cuarto del siglo pasado la autonomía de la ciencia procesal y de su categoría fundamental, el proceso, entendido como relación jurídica de carácter público caracterizada por la circunstancia de desenvolverse en una extensión temporal con la concurrencia de un representante del Estado (juez) y al menos dos sujetos interesados (las partes) en una decisión, que finalmente se debe obtener como resultado. ${ }^{1}$ La impor-

${ }^{1}$ Cf. CLÓVIS DO COUTO E SILVA, «Contribution a une histoire des concepts dans le droit civil et dans la procedure civile. (L'actualité de pensée d'Otto Karlowa et d'Oskar Bülow)», en: Quaderni fiorentini per la storia del pensiero giuridico moderno, n. 14, 1985, pp. 243ss., esp. p. 248, cuando señala la disposición de los juristas alemanes, en la segunda mitad del siglo XIX, a tratar los conceptos generales en cuanto parte integrante de una futura «Parte General del Derecho Civil». Por otro lado, no encuentra aceptación entre los procesalistas alemanes actuales el tipo de abordaje excesivamente abstracto y conceptualista, alejado de la realidad de los intereses concretamente envueltos en la determinación del sentido de normas e institutos procesales, tal como se practicó hasta mediados del siglo en curso en Alemania, y aún se practica bastante en los países latinos, por influencia de aquellos epígonos de un supuesto procesalismo científico. Véase, como caso paradigmático, F. v. HIPPEL, «Zur 'modernen, konstruktiven Epoche' der deutschen Prozessrechtswissenschaft», en: ZZP (= Zeitschrift für Zivilprozessrecht), n. 65, 1952, pp. 424ss., ahora también en la colección de ensayos de este autor Rechtstheorie und Rechtsdogmatik, Frankfurt am Main, 1964, pp. 357ss. La paradoja de la Escuela Histórica en su fase tardía, dominada por el pandectismo de B. WINDSCHEID, al haberse dedicado a un análisis formalista del derecho positivo desvinculándolo de condicionantes materiales de naturaleza histórica o política, es enfatizada por BÖCKENFÖRDE, Die Historische Rechtsschule und die Geschichtlichkeit des Rechts, Basel/Stuttgart, 1965, p. 24, 
tancia del procedimiento para el derecho es enfatizada en la influyente doctrina «pura» de Kelsen, cuando propone que se estudie no sólo la estática jurídica, cuyo objeto son las normas, sino también la teoría dinámica, que se ocupa de la conducta regulada por esas normas, responsable del proceso de ampliación y (auto)producción del derecho. ${ }^{2}$ En la misma época, las primeras décadas del siglo en curso, el procesalista James Goldschmidt realiza una «crítica del pensamiento procesal», proponiendo la consideración del proceso como momento regido por la dinámica inherente a esa misma noción, cuyo resultado puede perfectamente presentarse en desacuerdo con el que estática y abstractamente prevé el derecho material. ${ }^{3}$ Parecida es la formulación de Niklas Luhmann de la legitimidad obtenida a través del procedimiento, que hace dos décadas llamó la atención hacia la dimensión filosófica y política del fenómeno, en una investigación de carácter sociológico que lo tenía como objeto. ${ }^{4}$

Los finales de los años sesenta y principios de la década de los setenta marcan el acontecimiento de una renovación virtual de los estudios del derecho procesal, cuando se pasa a enfatizar la consideración del origen constitucional de los institutos procesales básicos. ${ }^{5}$ Proliferan, entonces, los aná-

passim; H. J. van EIKEMA HOLMES, Major Trends in the History of Legal Philosophy, Amsterdam/New York/Oxford, 1979, pp. 192ss.; F. WIEACKER, «Pandektenwissenschaft und industrielle Revolution», en Id., Industriegesellschaft und Privatrechtsordnung, Frankfurt a. M., 1974, pp. 55ss., esp. p. 61 y ss.

${ }^{2}$ Es reconocido de modo general que se debe a KELSEN la introducción de una perspectiva dinámica en el estudio del derecho, tal como se encuentra en su opus magnum, la Reine Rechtslehre, $2^{a}$ ed., Wien, 1960 (reimp. 1967). El carácter «procedimentalista» de su doctrina es referido por LUHMANN, en Legitimation durch Verfahren, Neuwied/Berlin, 1969, p. 11, nota 2.

${ }^{3}$ Cf. GOLDSCHMIDT, ob. ult. cit., cuyo subtítulo es «Eine Kritik des prozessualen Denkens», donde «crítica» debe ser entendida en el sentido epistemológico kantiano.

${ }^{4}$ Cf. LUHMANN, ob. cit. Otro sociólogo del derecho, en general menos conocido (discípulo, como el primero, de ARNOLD GEHLER), que llama la atención hacia la función social y específicamente jurídica, de fundamental importancia, desempeñada por el procedimiento, es HELMUT SCHELSKY. Para él, es de este último de donde viene la racionalidad propia del derecho, que no es puramente cognitiva, sino desplegada, pragmáticamente, hacia la orientación de la conducta, pues para él lo que importa no es la «verdad» (das «Wahre»), sino que se haga lo «cierto» (das «Richtige»). Cf. «Die jurisdische Rationalität», en: SCHELSKY, Die Soziologen und das Recht, Opladen, 1980, pp. 34ss., esp. pp. 35ss.; Id., «Nutzen und Gefahren der sozialwissenschaftlichen Ausbildung von Juristen», en: JZ (= Juristenzeitung), n. 13, 1974, pp. 410ss., esp. p. 412. En este aspecto, cuando enfatiza el carácter procedimental de la racionalidad jurídica, las ideas de SCHELSKY son corroboradas por la línea de pensamiento crítico, que él en vida tanto combatió, representada por HABERMAS (cf. loc. ult. cit.).

${ }^{5}$ Un fruto típico de esta tendencia, en Italia, es la obra de NICOLÒ TROCKER, Processo civile e costituzione. (Problemi di diritto tedesco e italiano), Milano, 1974. En Brasil hay diversos estudios en este sentido, como v. g. los de ADA PELLEGRINI GRINOVER. Las investigaciones reiteradas sobre el tema culminan con su promoción a objeto del VII Congreso Internacional de Derecho Procesal, en Wurzburg, RFA, en 1983. Cf. Eflektiver Rechtsschutz 
lisis de la conexión del proceso con la constitución, al punto de tomarse el derecho procesal por una especie de «derecho constitucional aplicado», como en cierta fecha formuló la corte constitucional alemana. Hasta el momento estos análisis se limitaron a propiciar esfuerzos en el sentido de realizar adaptaciones de la dogmática procesal a las exigencias de compatibilidad con los dictámenes de nivel constitucional relacionados directamente con el proceso. Estas son las garantías del llamado «debido proceso legal»: la independencia del órgano judicial, el derecho del interesado a tener acceso al juicio y a ser tratado con igualdad, etc. Todavía permanece inexplorada la vía que puede llevar a una completa reformulación del modo de concebir el proceso, con la tentativa de estructurarlo de acuerdo con los imperativos de un Estado social y democrático de derecho, como actualmente se configuran las sociedades políticas más desarrolladas, ya que se forman modernamente, sobre el influjo de las ideologías de fondo liberal, a partir del siglo pasado, pasando después por la influencia del autoritarismo predominante en el segundo cuarto del siglo en curso. ${ }^{6}$

\section{Proceso y constitución}

Lo que se pretende realizar a continuación no será tanto con respecto a la profundización de la relación del proceso con la constitución, tocando más de cerca el examen de la «otra cara de la moneda», es decir, la estrecha asociación entre constitución y proceso hoy en día, cuando éste se vuelve un instrumento imprescindible para la realización de aquélla.

Situémonos, así, frente a un doble movimiento en sentidos opuestos, o sea, uno hacia la materialización del derecho procesal, al condicionarlo a las determinaciones constitucionales; y al mismo tiempo, la procedimentalización o la «desmaterialización» del derecho constitucional, pues en la medida en que el proceso se muestre indispensable para la realización de la «Ley Mayor», lo es también para las menores u ordinarias. Es a clarificar este último aspecto que se destinan las consideraciones que presento.

La propuesta que se pretende sustentar en este punto es que la Constitución posee la naturaleza (también) de una ley procesal, así como institutos fundamentales del derecho procesal poseen estatuto constitucional y, por tanto, son (también) de naturaleza material. Esto presupone, de inmediato, que se afirme la distinción entre estos dos aspectos o dimensiones del dere-

und verfassungsmüssige Ordnung (Effectiveness of Judicial Protection and Constitutional Order), W. HABSCHEID (ed.), Bielefeld, 1983.

${ }^{6}$ Cf., sin embargo, RUDOLF WASSERMANN, Der soziale Zivilprozess. Zur Theorie und Praxis des Zivilprozesses in sozialen Rechtsstaat, Neuwied/Darmstadt, 1978, y K. E. SCHONFELD, Zur Verhandlungsmaxime im Zivilprozess und in den übrigen Verfahrensarten - Die Modifikation des Prozessrechts durch das Sozialstaatspostulat, Frankfurt a. M./Bern, 1981. 
cho, el material y el procesal, teniendo presente que no se trata propiamente de diferenciar ramas de la materia jurídica o de una división como la que separa derecho público y privado. Estamos, en verdad, ante nociones relacionadas, que se conceptúan una en función de la otra, y se exigen mutuamente. Materiales son las normas que contienen parámetros para realizar el control y ordenación de la conducta intersubjetiva por el derecho, en cuanto las normas procesales se ocupan directamente de esa realización, o sea, de la determinación de las condiciones para que estos parámetros sean aplicados concretamente.

Siendo así, se tiene que, desde una perspectiva formalista, según la cual lo que es propio de una constitución sería el establecimiento de normas para la elaboración e identificación de otras normas del orden jurídico en ella basado, podemos perfectamente clasificar las normas «por naturaleza» constitucionales como procesales. De hecho, todo el aspecto organizador, la distribución de competencias y de poderes entre las diversas esferas estatales, se reviste de un carácter procesal, al tratar de materia distinta de aquella que se considera derecho material, ya que no imponen directamente ningún patrón de comportamiento para ser asumido por los integrantes de la sociedad política. Por otro lado, no se puede dejar de considerar típicamente constitucional la fijación de ciertos modelos de conducta, por la distribución de derechos, deberes y garantías fundamentales, donde se va a encontrar la orientación para saber lo que se pretende alcanzar con la organización delineada en las normas de procedimiento.

El vínculo ligando constitución y proceso, que en la época actual -a la que ya solemos llamar «pos-moderna»- se muestra tan pronunciado, es una consecuencia natural del novum histórico, instaurado por la modernidad, en el terreno jurídico-social: la consagración de la victoria en la lucha por revolucionar la organización política mediante la redacción de un texto constitucional, i.e., «constitutivo» de un nuevo orden jurídico, un fenómeno bicentenario. El movimiento histórico de positivización del derecho, desencadenado por el ocaso de la autoridad basada en lo divino, implica la formación de un aparato burocrático cada vez mayor para implementar el orden jurídico. Tanto la legislación como la administración de la res publica y de justicia necesitan de formas procedimentales, dentro de las cuales puedan actuar atendiendo a los nuevos patrones de derecho, basados en la racionalidad y en el respeto al sujeto, portador de esta facultad. ${ }^{7}$

\footnotetext{
${ }^{7}$ Como acentúa VITTORIO DENTI, en «Dottrine del processo e riforme giudiziarie tra illuminismo e codificazioni», en: Diritto e potere nella storia europea. Atti del quarto Congresso internazionale della Società Italiana di Storia del Diritto (in onore di BRUNO PARADISI), vol. II, Firenze, 1982, pp. 883ss., «(L)a burocratizzazione della funzione giudiziaria risponde ad un programma politico di razionalizzazione del modus operandi degli organi giudi-
} 
Por tanto, la constitución asume el lugar de fundamento último del ordenamiento jurídico, una vez desaparecida la creencia en la fundamentación «sobrenatural» de un derecho de origen divino, y también la confianza en la «naturalidad» del derecho, que no precisa volverse objetivo por la positivación, por auto-evidente al sujeto dotado de racionalidad. Los valores fundamentales, sobre los cuales se erige ese ordenamiento, pasan a integrar ese mismo ordenamiento, al ser inscritos en el texto constitucional. ${ }^{8} \mathrm{La}$ consecución de esos valores, a su vez, requiere la intermediación de procedimientos, para que se tomen decisiones de acuerdo con ellos, siendo esos procedimientos, igualmente, establecidos con respecto a los valores. ${ }^{9}$ El proceso aparece, entonces, como respuesta a la exigencia de racionalidad, que caracteriza al derecho moderno. ${ }^{10}$

\section{El papel del proceso en el orden jurídico}

El cuadro hasta ahora descrito revela la faceta actual, eminentemente «autopoiética», del derecho como un sistema que regula a su propia (re)pro-

ziari che é uno degli aspetti fondamentali dell'iluminismo e che, d'altronde, corrisponde al sorgere del diritto amministrativo in censo moderno,(...). Alla razionalizzazione dell' apparato amministrativo corrisponde, sul piano scientifico, l'elaborazione della teoria dell'atto amministrativo, cosí come alla razionalizzazione dell'apparato giudiziario corrisponde l'elaborazione della categoria del procedimento» (pp. 886/887). Más adelante, Denti resalta que esta elaboración conceptual culmina en la «procesalización» de toda la actividad estatal: «Il massimo di razionalizzazione è dato, infatti, dall'applicazione della categoria del 'processo' a tutte le funzioni pubbliche, e quindi non soltanto alla funzione giudiziale, ma anche a quella amministrativa ed a quella legislativa» (p. 890).

${ }^{8}$ Cf. NIKLAS LUHMANN, Grundrechte als Institution, Berlin 1965, pp. 40 y ss., texto y notas 617 ; pp. 74 y ss., 182ss.

${ }^{9}$ Cf. DAVID RESNICK, «Due Process and Procedural Justice», en: Nomos (Yearbook of the American Society for Political and Legal Philosophy), n. 18, 1977, pp. 206ss., esp. pp. 217/219.

10 Cf. KLAUS EDER, «Prozedurale Rationalität», en: ARSoz (= Archiv für Rechtssoziologie), 7, 1986, pp. 1 ss., y los estudios críticos de HERBERT TREIBER, «Prozedurale Rationalität - eine "verfahrene Sache'?», ib. pp. 243ss., KARL-HEINZ LADEUR, id., pp. 265ss., y NIKOLAUS DIMMEL, id. ib., pp. 274ss.

${ }^{11}$ La teoría de los sistemas autopoiéticos fue desarrollada inicialmente por los biólogos chilenos HUMBERTO R. MATURANA, FRANCISCO E. VARELA y R. URIBE, en «Autopoiesis: The Organization of Living Systems, Its Characterisation and a Model», en: Bio System, n. 5, 1974, pp. $1897 \mathrm{ss}$. Su generalización para los sistemas sociales se debe a NIKLAS LUHMANN, que también la introdujo en la teoría del derecho, donde ha sido elaborada por GUNTHER TEUBNER en su doctrina del «Derecho reflexivo». Cf. TEUBNER/WILLKE, "Kontext und Autonomie. Gesellschaftliche Selbststeuerung durch reflexives Recht», ARSoz, 5, 1984, pp. 4ss.; TEUBNER, «Reflexives Recht», ARSP (= Archiv für Rechts- und Sozialphilosophie), 68, 1982, pp. 13ss.; Id., «Das regulatorische Trilemma», en: Quaderni fiorentini, n. 13, 1984, pp. 109ss.; Id., "Substantive and Reflexive Elements in Modern Law», en: Law \& Society Review, n. 17, 1983, pp. 239ss., y Autopoietic Law: A New Approach to Law and Society, 
ducción, por medio de procedimientos que él mismo instaura ${ }^{11}$. Dentro de este cuadro, no causa sorpresa el énfasis dado a la dimensión procesal del ordenamiento jurídico en recientes aportes teóricos ${ }^{12}$, ya que ante la cualidad de los problemas que depara la sociedad contemporánea, no se puede pretender encontrar (pre)escritas en ese ordenamiento las soluciones, que sólo se encuentran realmente ex post. De la misma manera, no se muestra satisfactoria la dogmática jurídica tradicionalmente practicada, pues presta atención predominantemente a los textos legales, para a partir de ellos reconstruir autorizadamente su sentido normativo, cuando el objeto de la ciencia jurídica no sería propiamente las normas, sino los problemas que a

TEUBNER (ed.), Berlin/New York, 1988, con contribuciones del propio TEUBNER, LUHMANN y numerosos teóricos europeos de las más variadas nacionalidades y norte-americanos. Para una evaluación crítica, véase, e.g., NOBERT REICH, «Reflexives Recht? Bemerkungen zu einer neuen Theorie von Gunther Teubner», en: FS (= Festschrift für); RUDOLF WASSERMANN, Neuwied/Darmstadt, 1985; ERHARD BLANKENBURG, «The Poverty of Evolutionism - A Critique of Teubner's Case for 'Reflexive Law'», en: Law \& Society Review, 18, 1984, pp. 273ss.; ARTHUR J. JACOBSON, «Autopoietic Law: The New Science of Niklas Luhmann», en: Mich. L. Rev. (= Michigan Law Review), n. 87, 1989, pp. 1647ss.; I. MAUS, «Perspektiven 'reflexiven Recht' im Kontext gegenwärtiger Deregulierungstendenzen», en: KJ, n. 19, 1986, pp. 390ss.; y las contribuciones de R. MUNCH y R NAHAMOWITZ en el ARSoz., n. 6, 1985. Por último, de TEUBNER, Recht als autopoietisches System, Frankfurt a. M., 1989.

${ }^{12}$ Se tiene en mente, como ejemplo típico, aparte de la recién mencionada doctrina de la «autopoiese», la teoría procedimental de WIETHÖLTER, la cual abordaremos en la conclusión del presente estudio. Para una tentativa reciente de repensar la categoría de «sujeto de derecho» incorporando elementos provenientes de ambas vertientes, consúltese REINER FREY, Vom Subjekt zur Selbstreferenz. Rechtstheoretische Überlegungen zur Rekonstruktion der Rechtskategorie, Berlin, 1989. También en el modelo desarrollado por K.-H. LADEUR, basado en la idea de «sopesamiento» (de intereses) -en alemán, Abwägung-, se llega a una «procedimentalización auto-referencial», que posibilita el (re)equilibrio y compatibilización de valores y comportamientos divergentes. Cf. LADEUR, "Abwägung - ein neues Rechtsparadigma», en: ARSP (= Archiv für Rechts- und Sozialphilosophie), n. 69, 1983, pp. 463ss.; Id. «Perspektiven einer post-modernen Rechtstheorie», en: Rechtstheorie (Zeitschrift für Logik, Methodenlehre, Kybernetik und Soziologie des Rechts), n. 16, 1985, pp. 383ss., cuyo texto en inglés aparece publicado en Autopoietic Law, cit., pp. 242ss. No menos dependiente de una reflexión sobre el proceso de realización del derecho se muestra la concepción teleológica que propone INGO MITTENZWEI, al revisar una antigua tradición filosófica a la luz de los nuevos desarrollos en la filosofía de la ciencia. Cf. Teleogische Rechtsverstandnis, Berlin, 1988. Finalmente, debe mencionarse el énfasis que en los últimos tiempos se viene dando al estudio de los «procesos de sopesamiento» (Abwägungsprozesse) de los valores jurídicos en la teoría del derecho escandinava. Cf. TORSTEIN ECKHOFF/NILS K. SUNDBY, Rechtssysteme, Berlin, 1988, pp. 105ss., passim.

${ }^{13}$ Una concepción en la cual los problemas jurídicos, y no las normas, son apuntados como el objeto de la ciencia del derecho, aparece, sin que haya recibido la debida atención, en MAX SALOMON, Grundlegung zur Rechtsphilosophie, $2^{\mathrm{a}}$ ed., Basel, 1925, pp. 23ss. Esta posición se ve bastante fortalecida con el predominio del paradigma de la «jurisprudencia de intereses» en la dogmática jurídica alemana, así como por la aparición de una teoría tópica del derecho, debida a THEODOR VIEHWEG, que cierra la «Introducción» de su Topik und jurisprudenz calificando a la dogmática jurídica (Jurisprudenz) como «un procedimiento especí- 
ellas cabe solucionar ${ }^{13}$. Y para eso importa más que todo el examen de las situaciones concretas en las que los intereses envueltos se manifiestan y (eventualmente) entran en conflicto; ahí radica la importancia de normas procedimentales que regulen el modo de atender a esos intereses, con una solución que no se encuentra ya previamente establecida.

La concepción del orden constitucional como un proceso, en el cual participan los defensores de interpretaciones muy diversas cuando de la concreción de ese orden se trata, se contrapone a la concepción estática de ese mismo orden como ya establecido, y está en sintonía con la mencionada orientación en teoría del derecho que lo concibe como autopoiético. $^{14}$

\section{Proceso y teoría de la justicia}

De lo expuesto entendemos que las direcciones más diversas en teoría y filosofía del derecho están apuntando, actualmente, en el sentido de una reflexión sobre los procedimientos a través de los cuales se realiza, con la preocupación de establecer parámetros de justicia y racionalidad para balizarlos; justicia y racionalidad que, a su vez, el derecho tendrá que atender por medio de aquellos procedimientos. La cuestión de la justicia, por ejemplo, por la cual volverán a discutir recientemente los filósofos, en el ámbito de una rehabilitación de la filosofía práctica, es tratada en una de las más importantes doctrinas del momento, la del filósofo norteamericano John Rawls, como indisolublemente ligada al procedimiento de su realización,

fico de suscitar problemas, debiendo este procedimiento ser entendido como el objeto de la ciencia

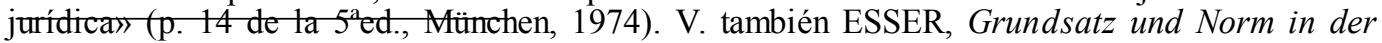
richerlichen Forbildung des Privatrechs. Rechtsvergleichende Beiträge zur Rechtsquellen und Interpretationslehre, $2^{\mathrm{a}}$ ed., Tübingen, 1964, p. 6, texto y nota 13, pp. 48ss.

${ }^{14}$ La constitución es concebida por HÄBERLE como un proceso, abierto a la participación pluralista de representantes de las más variadas interpretaciones. Cf. «Die offene Gesellschaft der Verfassungsinterpreten. Ein Beitrag zur pluralistischen und 'prozessualen' Verfassungsinterpretation», en: JZ, n. 30, 1975, pp. 297ss., ahora también en: Id., Verfassung als offerntlicher Prozess, Berlin, 1978, pp. 155ss., con un registro de la discusión suscitada por el ensayo, recibido con entusiasmo por unos y repudiado por el positivismo constitucional, con BÖCKENFÖRDE al frente (pp. 180ss.). Consúltese, además, en el mismo volumen, los estudios inéditos «Verfassungsinterpretation als öffentlicher Prozess - ein Pluralismuskonzept», pp. 121ss., y «Verfassungsinterpretation und Verfassungsgebung», pp. 182ss., esp. pp. 189ss. También en EE.UU. surge a comienzos de la década pasada una serie de teorías constitucionales de carácter procesal, como las de J. CHOPER, en Judicial Review and National Political Process, y J. H. ELY, en Democracy and Distrust. A Theory of Judicial Review. Cf. el examen crítico de R. D. PARKER, «The Past of Constitutional Theory - and its Future», Ohio State Law Journal, 1981, pp. 223 ss., y la defensa de HABERMAS, loc. cit., pp. 15ss., el cual se alinea entre los simpatizantes de tales teorías, como muestra su estudio «Volkssouveranität als Verfahren», en: MERKUR (Deutsche Zeitschrift für europäisches Denken), n. 43, 1989, pp. 465ss. esp. pp. 475ss., ahora incluido en Faktiztät und Geltung. Otro filósofo de gran prestigio en la actualidad, que suscribiría la teoría del carácter procedimental de la constitución, es 
donde la idea que va a buscar en Brian Barry, de una «Justicia Procedimental» (procedural justice. ${ }^{15}$ Ésta puede presentarse en dos formas: una pura o perfecta, cuando se trata de un procedimiento «estrictamente técnico», como el de distribuir una tarta entre varias personas (el ejemplo es de Rawls); y otra imperfecta, que es la revestida de mayor importancia práctica, de la cual los procesos judiciales (penal, civil, etc.) son un ejemplo. ${ }^{16}$

También la tematización del derecho natural, que hoy se coloca en un terreno menos jurídico que moral, asume una formulación, en los condiscípulos de Arthur Kaufmann como Winifried Hassemer y Gunther Ellscheid, como dotado de carácter eminentemente procedimental: para ellos lo que importa no es tanto establecer principios con un contenido moral, sino principios procedimentales (prozedurale Prinzipien), para guiar interesados en hacer asertivas dotadas de contenido iusnaturalista. ${ }^{17}$

JOHN RAWLS, quien la concibe, idealmente, como un procedimiento balizado por principios de justicia, en el cual se conformarían las fuerzas políticas, responsables de la producción legislativa. En sus propias palabras: «Ideally ajust constitution would be ajust procedure arranged to insure a just outcome. The procedure would be the political process governed by the constitution, the outcome the body of enacted legislation, while the principles of justice would define an independent criterion for both procedure and outcome». A Theory of Justice, Oxford, 1972, p. 197.

${ }^{15}$ Cf. BARRY, Political Argument, London, 1965, cap. VI, y RAWLS, ob. cit., pp. 83ss.

${ }^{16}$ En este punto, se puede aprovechar para cuestionar la opinión, extremamente difundida aún en una procesalística actualizada como es la brasileña, según la cual el ordenamiento procesal es un «instrumento eminentemente técnico»; la expresión es de ALFREDO BUZAID, autor del proyecto del Código Procesal Civil vigente, en su «exposición de motivos», ahora presentada como prefacio a Il processo civile brasiliano, N. PICARDI/A. GIULIANI (eds.), Rimini, 1988, p. 1. Que el derecho procesal sería un «Derecho técnico» lo postulaba ya FRIEDRICH STEIN en el prefacio a la $1^{\mathrm{a}}$ ed. de su Grundrißes des Zivilprozessrechts und Konkursrechts (1921). Al defender esa idea, el autor del proyecto de nuestro CPC no pretendió excluir el proceso de la dimensión axiológica, ya que lo concibe «permeable a principios rigurosamente éticos» (id. ib., p. 2). Tampoco STEIN abandonó por completo las reflexiones iusfilosóficas, especialmente sobre la naturaleza de la ejecución forzosa, como recuerda FRIEDRICH WEBER, en «Methodik des Prozessrechts», en: Studiunt Generale (Zeitschrift für die Einheit der Wissenschaften im Zusammenhang ihrer Begriffsbildungen und Forschungsmethoden), n. 13, 1960, pp. 183ss., 183, texto y nota 1. Por otro lado, es hoy indudable la influencia que la ideología ejerce sobre el proceso. Cf., v.g., ERNST J. COHN, «Zur Ideologie des Zivilprozessrecluts», en: Erinnerungsgabe für Grünhut, Marburg, 1965, pp. 31 ss.; DIETER LEIPOLD, «Zivilprozessrecht und Ideologie», JZ, 37, 1982, pp. 441ss.; TARELLO, «L'opera di Giuseppe Chiovenda nel crepuscolo dello stato liberale, en: Id. (ed.), Materiali per una storia della cultura giuridica, T. III, vol. I, Bologna, 1973, pp. 691ss.; MAURO CAPPELLETTI, Processo e Ideologie, Bologna, 1969.

${ }^{17}$ Una concepción procedimental del Derecho Natural fue presentada por HASSEMER en el seminario que impartió en el Curso de Maestría de la Facultad de Derecho de la Universidad Federal de Ceará, en 1984. Cf., de ELLSCHEID, «Das Naturrechtsproblem. Eine Systematische Orientierung», en: A. KAUFMANN/W. HASSEMER (eds.), Einführung in der Rechtsphilosophie und Rechtstheorie der Gegenwart, 4ª ed., Heidelberg, 1985, pp. 125ss., esp. pp. 158ss. 
Así es que tanto el iusnaturalismo como también aquella otra vía que tradicionalmente se le contrapone, por privilegiar el análisis del derecho tal como se presenta en cuanto dato positivo, y el llamado positivismo lógico, terminan por consagrarse, actualmente, a los problemas en torno a la argumentación jurídica. Ya que es allí donde se define tanto la cuestión de la justicia -enfatizada por los defensores de aquella primera vertiente-, como igualmente la cuestión de la racionalidad -la que más interesa a los positivistas- de los actos practicados para cumplir el derecho. Los estudiosos de tendencia analítica pasan así a ocuparse no sólo de las normas jurídicas que se encuadran en el esquema "previsión normativa/consecuencia jurídica», las llamadas «reglas», sino también de aquellas otras normas sobre las que se erige el ordenamiento jurídico en su totalidad -sin dejar de integrarlo-, que son sus principios fundamentales. Es en este contexto donde se sitúa la reciente doctrina de Robert Alexy, con su modelo de argumentación jurídica e interpretación constitucional basado en la distinción entre reglas, principios y procedimiento (Regeln/Prinzipien/Prozedur-Model). 
DOXA 21-II (1998) 